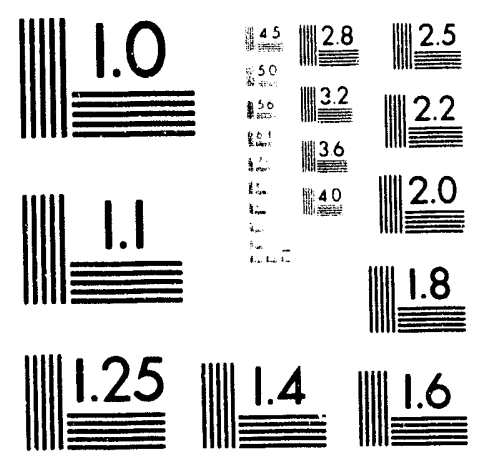



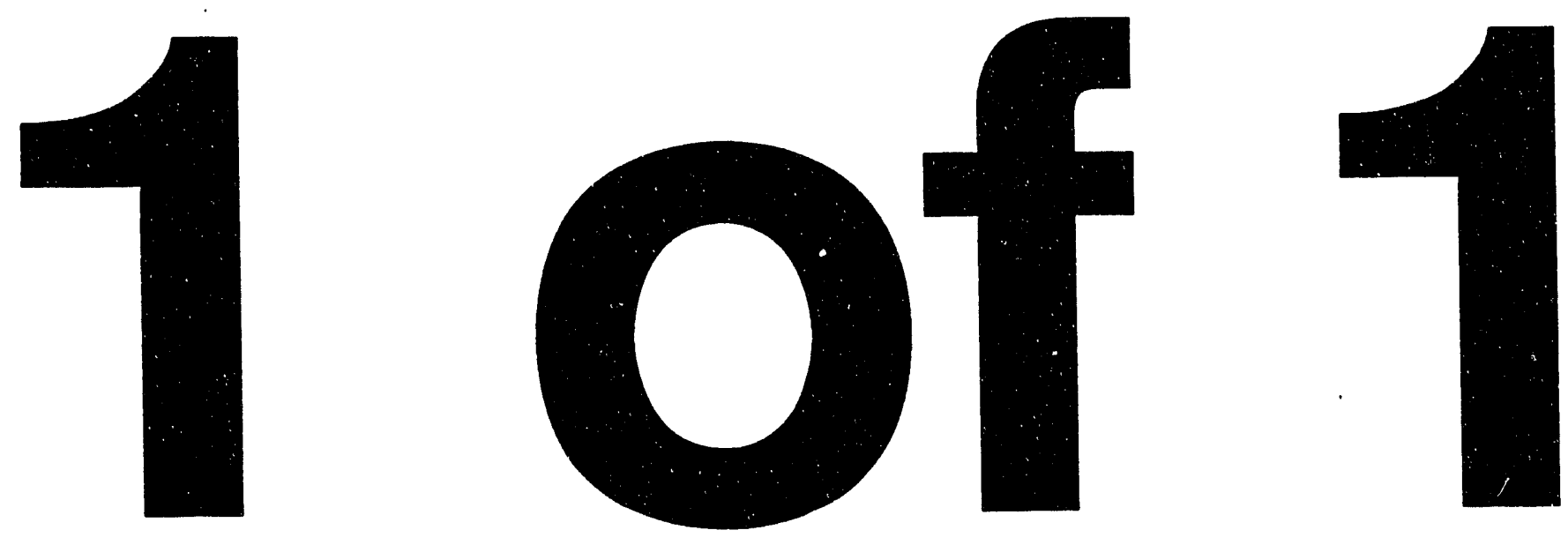


\title{
Analysis of Higher Order Optical Aberrations in the SLC Final Focus using Lie Algebra Techniques."
}

\author{
N. J. Walker, J. Irwin, M. Woodley \\ Stanford Linear Accelerator Center, Stanford University, Stanford, California 94309
}

\section{Abstract}

The SLC final focus system is designed to have an overall demagnification of $30: 1$, with a $\beta$ at the interaction point $\left(\beta^{*}\right)$ of $5 \mathrm{~mm}$, and an energy band pass of $\sim 0.4 \%$. Strong sextupole pairs are used to cancel the large chromaticity which accrues primarily from the final triplet. Third-order aberrations limit the performance of the system, the dominating terms being $\mathrm{U}_{1266}$ and $\mathrm{U}_{3466}$ terms (in the notation of $\mathrm{K}$. Brown). Using Lie Algebra techniques, it is possible to analytically calculate the size of these terms, in addition to understanding their origin. Analytical calculations (using Lie Algebra packages developed in the Mathematica language) are presented of the bandwidth and minimum spot size as a function of divergence at the interaction point (IP). Comparisons of the analytical results from the Lie Algebra maps and the results from particle tracking (TURTLE) are also presented.

\section{INTRODUCTION.}

The SLC final focus design consists of two telescopes, each with point to point focusing (phase advance of $\pi$ radians), separated by a $2 \pi$ radian identity module with a large dispersion function which facilitates the second (optical) order chromaticity correction using strong sextupoles: figure 1 shows the optics. The chromatic correction scheme and overall telescopic

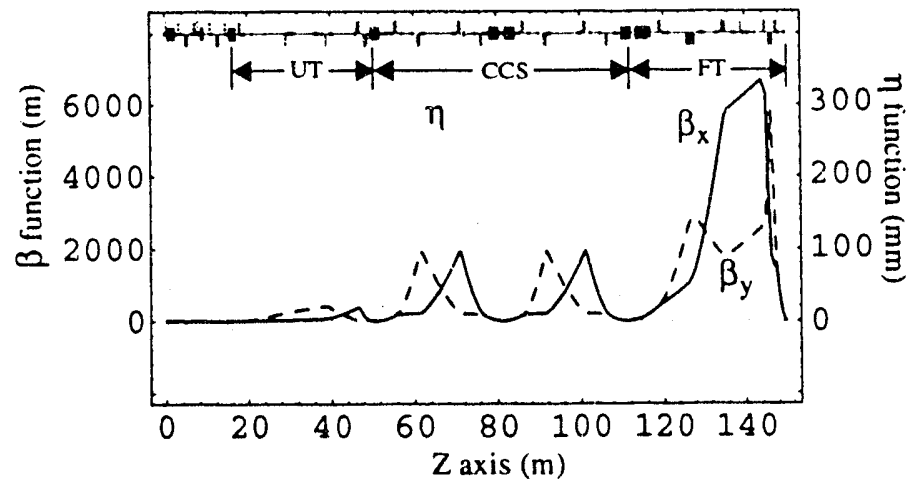

Figure 1. Optics functions for the SLC final Focus

design are essentially that proposed by Brown[1] and is well documented in the literature[2]. The predominant source of chromaticity is the final triplet, for which the correction scheme consists of two -I pairs of sextupoles placed in such a position that the dispersion function is symmetric: such an arrangement exactly cancels the unwanted geometrics, leaving

\footnotetext{
- Work supported by Department of Energy contract DE-AC03-76SF00515 Presented at the Partite Accelerator
}

only the desired chromatic terms.

Although the second order optical aberrations are exactly cancelled, the bandwidth of the system is still limited by third (optical) order aberrations. Brown[1] showed that the dominant remaining aberration is a high order chromaticity $\left(U_{1266}\right.$ and $\mathrm{U}_{3466}$ in the TRANSPORT[3] notation). Using powerful Lie Algebra techniques, it is possible to analyze the individual aberrations and understand their origins. In this report, an analysis of the important aberrations using Lie Algebra tools developed in the Mathematica[4] language is presented.

\section{LIE ALGEBRA TECHNIQUES.}

Lie Algebra techniques as applied to charged particle optics have been extensively covered elsewhere[5,6,7]. Here extensive use of Lie Algebra methods developed by Irwin[6] is made, of which a good review can be found in the thesis by Roy[7]. Only a brief introduction to the terminology and general philosophy of the methods is given here: the reader is referred to the literature for a more detailed description of the subject.

In the following subsections the important Lie Algebra tools are introduced which will later be used to analyze the aberrations. In the following discussions, $x^{\prime} \equiv \partial x / \partial s$, is used rather than the conjugate momentum $p_{x}$, as this is more in keeping with the traditional matrix theory of Brown[2].

\section{A. Hamiltonians.}

The starting point of the calculation is the formulation of the perturbed Hamiltonian for the elements (magnets) of concem, and evaluating them at the non-perturbed, linear phase space coordinates at that element; this type of calculation is referred to as an interaction representation.

For the present analysis, two elements are of interest:

$$
\begin{array}{ll}
\text { Chromatic Quadrupole: } & -\frac{1}{2} K_{Q} \bar{\delta}\left(x^{2}-y^{2}\right) \\
\text { Sextupole: } & \frac{1}{3 !} K_{S}\left(x^{3}-3 x y^{2}\right)
\end{array}
$$

where $K_{Q}$ and $K_{S}$ are the integrated quadrupole and sextupole strength respectively, $\bar{\delta}$ is given by $\delta /(1+\delta)$, where $\delta$ is the fractional momentum deviation $\Delta P / P_{O}$, and $x$ and $y$ are the local linear phase space coordinates. The Hamiltonians given in (2) and (3) represent thin lens kicks. To include the effects of thick (lumped) elements, it is necessary to integrate the Hamiltonian over the length $(L)$ of the magnet: 


$$
H_{\text {thick }}\left(x_{c}, x^{\prime}, y_{c}, y_{c}^{\prime}\right)=\int_{-L / 2}^{L / 2} H_{\text {thin }}(x(s), y(s)) d s
$$

where $\left(x_{c}, x_{c}^{\prime}, y_{c}, y_{c}^{\prime},\right)$ represent the coordinates at the center of the magnet. $x(s)$ and $y(s)$ are given by the linear optics of the element[5].

\section{B. Poisson Brackets}

At the heart of the Lie Algebra approach is the Poisson Bracket (PB). The PB of any two functions of phase space $f$ and $g$ is given by

$$
[f, g]=\frac{\partial f \partial g}{\partial x \partial x^{i}}-\frac{\partial f \partial g}{\partial x^{\prime} \partial x}
$$

Hamilton's equations of motion can be represented using $\mathrm{PB}$ as

$$
\begin{aligned}
\frac{\partial x}{\partial t} & =[-H, x] \\
\frac{\partial x^{\prime}}{\partial t} & =\left[-H, x^{\prime}\right]
\end{aligned}
$$

where $H$ is the Hamiltonian.

One important property of poisson brackets is that they remain invariant under a symplectic transformation; this property allows the transformation of the local coordinates in the Hamiltonians to any point in the lattice using the linear Green's functions ( $R$ matrix elements). In the beam optics calculations that follow, use is made of the invariance property to transform all the coordinates to the interaction point (IP) of the final focus.

\section{Beam line Representation: $C B H$ theorem.}

If $H_{i}$ represent the Hamiltonians for the magnetic elements in the beam line, then the line can be represented in the exponential Lie notation as

$$
e^{:-H_{1}:} e^{:-H_{2}:} e^{:-H_{3}:} \ldots e^{:-H_{N}:}
$$

where the $H_{i}$ are placed in the order they appear in the beamline. The total effective Hamiltonian for the system can be calculated using the Cambell-Baker-Hausdorf $(\mathrm{CBH})$ theorem:

$$
H_{T}=\sum_{i=1}^{N} H_{i}-\frac{1}{2} \sum_{i=1}^{N} \sum_{j=1}^{i}\left[H_{j}, H_{i}\right]+\ldots
$$

Each PB term in (7) represents higher and higher orders in the Hamiltonian. In the work reported here, the $H_{i}$ are the thirdorder Hamiltonians given in (1) and (2), so the first PB in the $\mathrm{CBH}$ theorem generates fourth order terms; this is sufficient for the analysis of the SLC final focus.

In the exponentiated form, the Hamiltonian becomes a generator for a map:

$$
x \rightarrow e^{i-H_{T}:} x=x+\left[-H_{T} x\right]+\frac{1}{2}\left[-H_{T}\left[-H_{T}, x\right]\right]+\ldots
$$

For the analysis of the final focus, $H_{\mathrm{T}}$ is a polynomial expressed in the linear phase space coordinates at the IP. Each monomial in $H_{\mathrm{T}}$ represents a unique aberration, the effect of which can be approximated independently by taking the first PB term in (8):

$$
\Delta x \approx\left|-H_{7}, x\right|=\frac{\partial H_{T}}{\partial x^{\prime}}
$$

Equation 9 can be used to estimate the effect of each individual monomial in the Hamiltonian on the IP spot size. It is important to note, however, that when making an exact third- (optical) order calculation, it is important to also include the second $\mathrm{PB}$ term in (8), as this will contribute to third-order terms in the map.

\section{Mathematica TOOLS.}

Mathematica[4] is a powerful language for doing symbolic computations. Since the Lie Algebra techniques presented in the previous section involve the manipulation of polynomials, Mathematica is ideally suited to the task. Several Mathematica packages have been developed to enable Lie Algebra analysis of beamlines, collectively referred to as LAMA (Lie Algebra Mathamatica Analysis) packages: they are

LAMA TransportDefinition`.

A package containing a framework for the definition of inagnetic elements and beamlines, together with tools for manipulating and modifying them.

\section{LAMA LinearOptics".}

A package for doing simply linear optics, such as calculation of tables of $\mathrm{R}$ matrices for a given beamline.

\section{LAMA'PoissonBracket'.}

Implements $\mathrm{PB}$ of predefined phase space coordinates.

\section{LAMA HamiltonianOptics`.}

Contains all the definitions of the Hamiltonians for known magnetic elements, and performs thick lens integration (equation 3). Also contains definitions for Lie algebra tools and several high level analysis tools and manipulation tools for polynomials.

\section{LAMA'Expectation Value .}

A package for calculating high order moments, and expanding them in terms of second order moments of given distributions.

Since Mathematica is a symbolic language, parameters such as magnet strengths can be left as symbols to allow fitting.

\section{RESULTS OF SLC FINAL FOCUS ANALYSIS.}

Figure 2 shows a curve of $\sigma_{x}^{*}$ and $\sigma_{y}^{*}$ as a function of linear beam divergence at the IP $\left(\theta^{*}\right)$. The solid curve is the result of a symbolic third (optical) order map generated using the Mathematica Lie Algebra tools (essentially equations 7 and 8), while the dots represent the results of TURTLE[8] simulations: the analytical results generated by Mathematica are in good agreement with the si nulations. It is important to emphasize the difference between simulation and symbolic calculations 


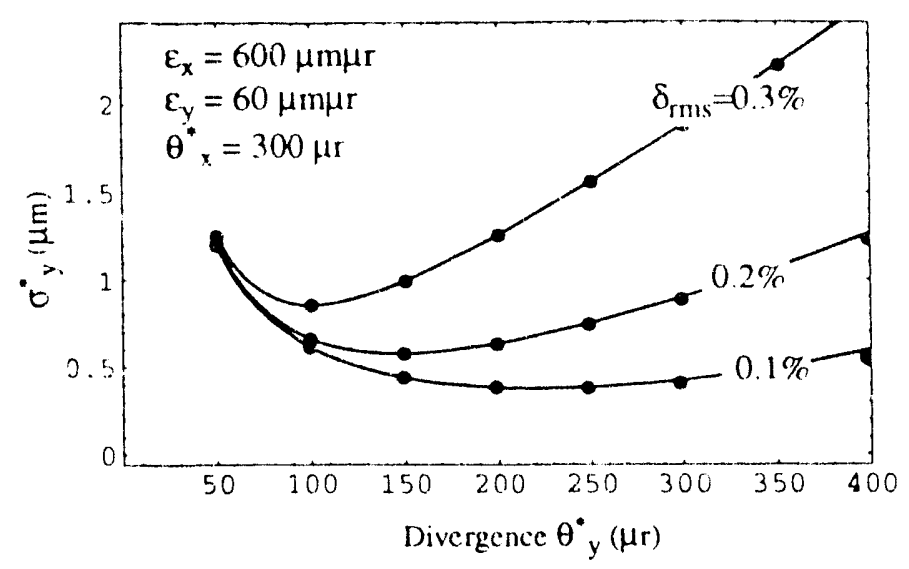

Figure 2. Vertical IP spot size as a function of divergence for various heam energy spreads $\left(\delta_{\mathrm{rn} s}\right)$. Solid lines represent symbolic Mathemat ica calculation, dots represent TURTLE simulations.

the Mathematica results are analytical (symbolic) formulae of the from $\sigma^{*}{ }_{y}=\sigma^{*}{ }_{y}\left(\varepsilon_{x}, \varepsilon_{y}, \theta^{*}{ }_{x} \theta^{*}{ }_{y} \delta_{r m . s}\right)$, and can be manipulated accordingly.

Table 1 gives the results of a term by term analysis of the total Hamiltonian with respect to the vertical IP spot size $\left(\sigma^{*}{ }^{*}\right)$.

Table 1. Most significant aberrations to $\sigma^{*}$ in order of contribution.

\begin{tabular}{cccc}
\hline Monomial & $\begin{array}{c}\text { TRANSPORT } \\
\text { notation }\end{array}$ & $\begin{array}{c}\text { Cocficient } \\
\text { (meters) }\end{array}$ & $\begin{array}{c}\% \text { of total } \\
\sigma^{2}\end{array}$ \\
\hline$y^{2} \delta^{2}$ & $\mathrm{U}_{3466}$ & 229.5 & 86 \\
$x^{\prime} y^{\prime 2} \delta$ & $\mathrm{U}_{1446} / \mathrm{U}_{3246}$ & 817.5 & 6 \\
linear & & & 3 \\
$x^{\prime 2} y^{\prime 2}$ & $\mathrm{U}_{1244} / \mathrm{U}_{3224}$ & -2861.7 & 2.2 \\
$x^{\prime} y^{\prime} \delta^{2}$ & $\mathrm{U}_{1466} / \mathrm{U}_{3266}$ & 55.5 & 1.9 \\
\hline
\end{tabular}

By far the most dominant aberration is the $y^{2} \delta^{2}$, which in TRANSPORT notation is the afore mentioned $\mathrm{U}_{3460}$ term. One possible method of determining the origin of such a term is by identifying which PBs in the second term of the CBH rontribute to the total coefficient. Replacing the double sum in (7) by a matrix $A_{i j}=\left[H_{i}, H_{j}\right]$ of polynomials, whose upper diagonal elements are zero, one can easily extract the coefficient of the $y^{\prime 2} \delta^{2}$ term in each of the polynomials in $A$. Figure 3 shows a three dimensional bar chart representing the contributions from the $A_{i j}$ polynomials to the total $y^{\prime 2} \delta^{2}$ term.

Figure 3 immediately reveals that the largest source of the $y^{\prime 2} \delta^{2}$ aberration comes directly from an interaction of the sextupoles and the triplet. Further investigation shows that the $Y$ sextupoles are not exactly $\pi$ radians in phase away from the triplet, and that this phase error gives rise to a small chromaticity term $\xi_{2 y} \delta$. Interaction of the $2 y^{\prime} \delta$ aberration with the strong chromaticity of the triplet $\left(\alpha y^{\prime 2} \delta\right)$ results in the $y^{\prime 2} \delta^{2}$ term:

$$
\left[\xi y y^{\prime} \delta, \alpha y^{\prime 2} \delta\right]=2 \alpha \xi y^{\prime 2} \delta^{2}
$$

Having identified the source of the aberration, it is now possible to design a modification to the linear optics to correct the phase error of the sextupoles, and thus reduce the magni-

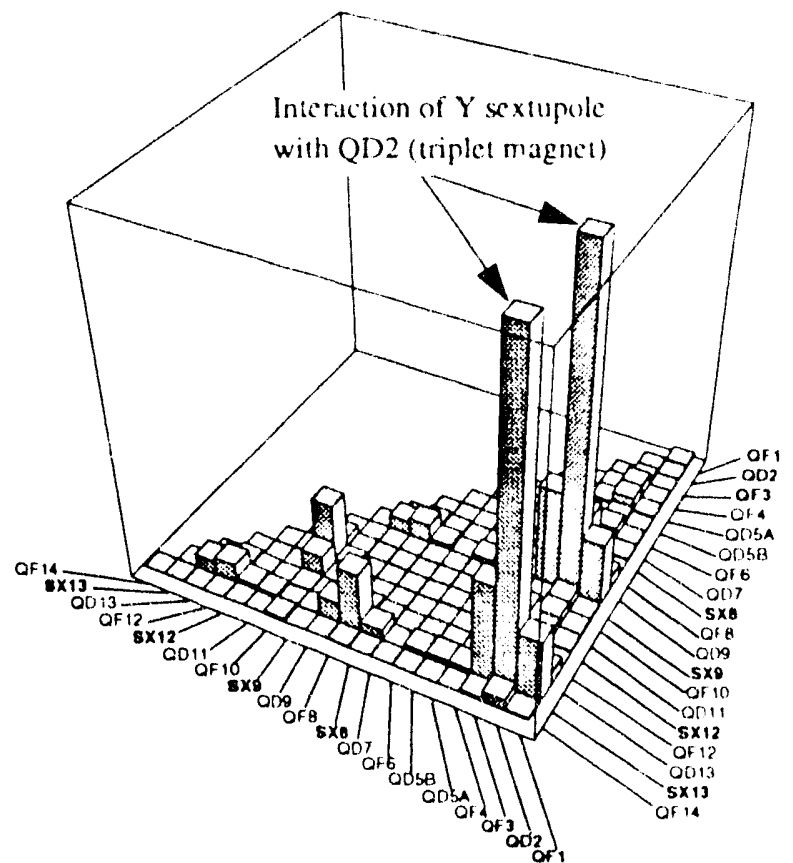

Figure 3. Coefficient of $y^{\prime 2} \delta^{2}$ monomial (arb. units) in the interaction polynomial $\left[H_{1}, H_{j}\right]$ for the CCS and final telescope magnets.

tude of the aberration [9].

\section{REFERENCES}

11] K. Brown, "A Conceptual Design of Final Focus Systems for Linear Colliders", SLAC-PUB-4159, (1987).

[2] J. Murray, K. Brown, and T. Fieguth, "The Compleled Design of the SLC. Final Focus System", SLAC-PI/B4219 (1987).

[3] K. Brown, "A First- and Second-Order Matrix Theory for the Design of Beam Transport Systems and Charged Particle Spectrometers", SLAC-75 or Advances in Particle Physics 1, 71 (1967).

[4] S. Wolfram, "M'athematica: A System for Doing Mathematics by Computer", Second Edition, Addison-Wesley Publishing Cornpany (1991).

15] A. Dragt, F. Neri, G. Rangarajan, D. Douglas, L. Healy, and R. Ryne, "Lie Algebaic Treatment of Linear and Nonlinear Beam Dynamics", Ann. Rev. Nucl. Parr. Sci. 38, 455 (1988).

16] J. Irwin, "The Application of Lie Algebra Techniques to Beam Transport Design", Nuc. Inst. and Methods, A298, 460 (1990).

[7] G. Roy, "Analysis of the Optics of the Final Focus Test Beam using Lie Algebra based Techniques", PhD thesis, SLAC, (1992).

[8] D. Carey, K. Brown, and C. Iselin, "TURTLI: A Computer Program for Simulating Charged Particle Beam Transport Systems, Including Decay Calculations", SLAC-246, and Fermilab PM-31 (1982).

[9] N. Walker, J. Irwin, R. Helm, and M. Woodley, "ThirdOrder Corrections to the SLC Final Focus", these proceedings (1993). 

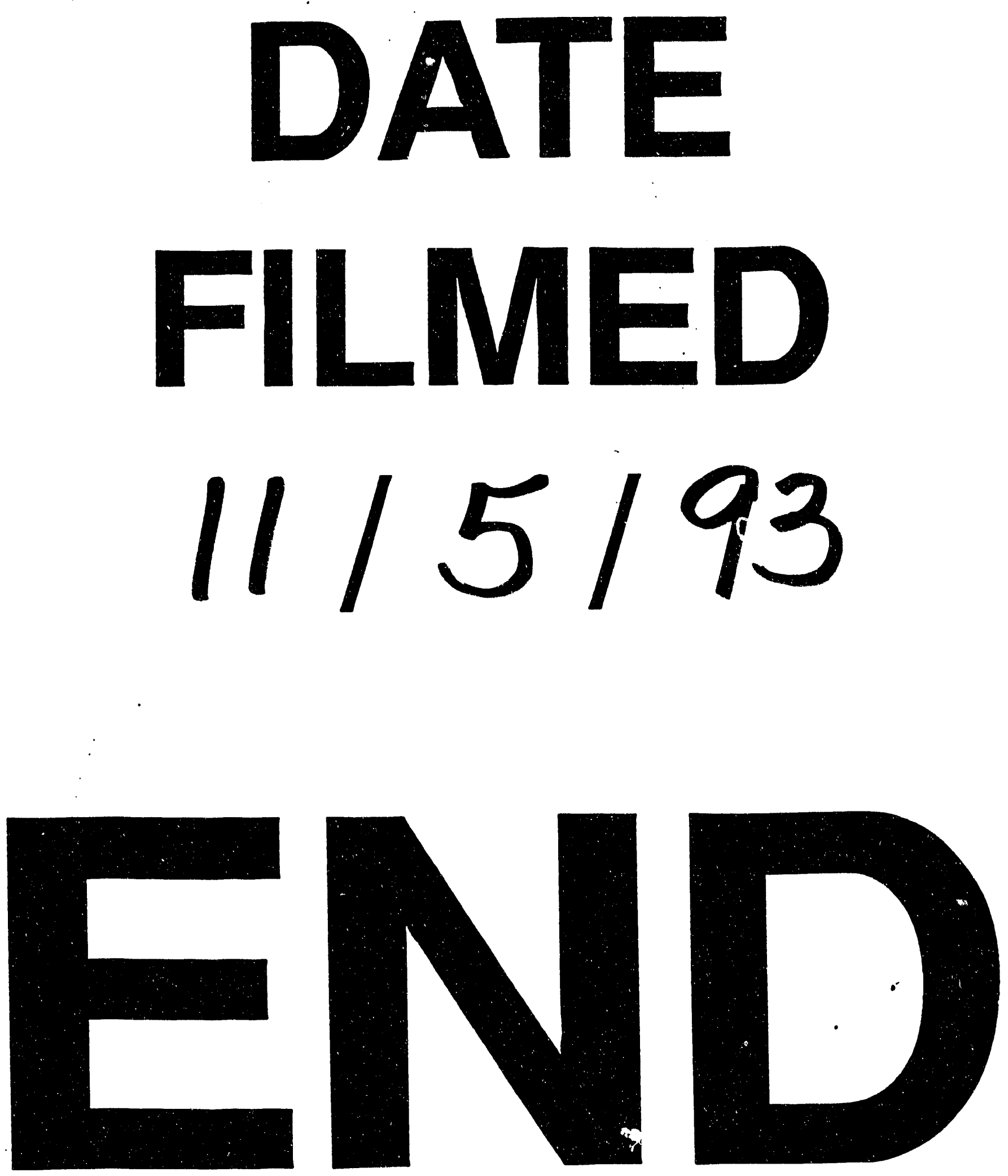
